\title{
INDECOMPOSABLE REPRESENTATIONS OF GROUPS WITH A CYCLIC SYLOW SUBGROUP
}

\author{
BY \\ G. J. JANUSZ
}

1. Introduction. D. Higman proved in [6] that the group algebra $K(G)$ of a finite group $G$ over an algebraically closed field $K$ with characteristic $p$ has only a finite number of inequivalent indecomposable modules if and only if the $p$-Sylow subgroups of $G$ are cyclic. Higman also gave an estimate of the number of such modules. In [7] Kasch, Kneser, and Kupisch gave a sharper upper bound and conditions on $G$ under which the bound would be attained. In this paper we apply J. Green's notion of a vertex and source to give a formula for the exact number of indecomposable $K(G)$-modules.

In $\S 3$ we consider the special case $G=L F(2, p)$ and $K$ of characteristic $p$. Starting with maximal submodules of the principal indecomposable $K(G)$-modules we construct all the indecomposable $K(G)$-modules. The idea is to form certain subdirect sums of these modules. In the Appendix we consider the subdirect sum of two modules over an arbitrary ring, and give a test to determine when the subdirect sum is indecomposable.

2. The counting formula. Let $G$ be a finite group and $K$ an algebraically closed field of characteristic $p$. Recall that a $p$-subgroup $Q$ of $G$ is called a vertex for an indecomposable $K(G)$-module $M$ if $M$ is a direct summand of the induced module $L^{G}$ for some indecomposable $K(Q)$-module $L$, and $Q$ is a minimal such subgroup. In this situation $L$ is called a source for $M$. Whenever $M$ has vertex $Q$ and $H$ is a subgroup of $G$ containing $Q$, then $M$ is a direct summand of $\left(M_{H}\right)^{G}$. In this situation the vertex and source of $M$ are determined up to conjugates (Green [6]). Any further terminology and notation can be found in Curtis and Reiner [3].

From now on we assume $G$ has a cyclic $p$-Sylow subgroup. Let $Q$ be a $p$-subgroup of $G$ and $N$ its normalizer. The first proposition was proved by Green in [5] but the additional hypothesis of this section enables us to give a very short proof.

Proposition. There is a one-to-one correspondence between the set $\{M\}$ of indecomposable $K(G)$-modules with vertex $Q$ and the set $\{W\}$ of indecomposable $K(N)$-modules with vertex $Q$. If $M$ corresponds to $W$ then $M$ is a direct summand of $W^{G}$ and $W$ is a direct summand of $M_{N}$.

Received by the editors March 28, 1966. 
Proof. Let $M_{N}=W_{1} \oplus \cdots \oplus W_{t}$ be a decomposition of $M_{N}$ by indecomposable $K(N)$-modules. Because $M$ is a component of $\left(M_{N}\right)^{G}, M$ must be a component of $W^{G}$ for $W$ one of the $W_{i}$. We show this $W$ is unique. The Mackey formula (44.2) of [3] gives

$$
\left(W^{G}\right)_{Q}=\sum_{D} W(D)
$$

where the sum is taken over the distinct double cosets $D=Q z N$ for $z$ in $G$, and $W(D)=\left\{W_{Q \cap z N z^{-1}}\right\}^{Q}$. We can have $Q=Q \cap z N z^{-1}$ if and only if $z^{-1} Q z \subseteq N$. Because the $p$-Sylow subgroup of $G$ is cyclic, this can happen if and only if $z^{-1} Q z=Q$; that is $z \in N$. Hence there is exactly one $D$ for which $W(D)=W_{Q}$ and for all others, $W(D)$ is a sum of modules with vertex properly contained in $Q$. But now $M$ is a direct summand of $W^{G}$ so $M_{Q}$ is a direct summand of $\left(W^{G}\right)_{Q}$. Moreover $W_{Q}$ is a direct summand of $M_{Q}$. Hence all other summands of $M_{Q}$ have vertex properly contained in $Q$. In particular all $K(N)$-summands of $M_{N}$ other than $W$ have vertex $\neq Q$. Hence $W$ is unique.

Now we want to count the number of nonisomorphic indecomposable $K(G)$ modules. We shall proceed in the following way: For a $p$-subgroup $Q$ we first count the number of indecomposable $K(G)$-modules with vertex $Q$. By Proposition 1 it is sufficient to count the number of indecomposable $K(N(Q))$-modules with vertex $Q$. To accomplish this we count the number of nonisomorphic direct summands of the induced module $L^{N}$ when $L$ is an indecomposable $K(Q)$-module with vertex $Q$. The last step is to count the number of choices for $L$.

Let $Q$ be a $p$-subgroup with generator $y \neq 1$. The ideals $K(Q)(y-1)^{i}, i=0, \ldots$, $|Q|-1$, give a full set of isomorphism types of indecomposable $K(Q)$-modules. If $L$ is such an ideal, the induced module $L^{N}$ can be identified with the left ideal $K(N) L$. For any $x \in N$ we have $x^{-1} L x=L$ because $x^{-1} K(Q) x=K(Q)$ and the ideals of $K(Q)$ are linearly ordered. Thus $K(N) L$ is a two-sided ideal. Let $\left\{e_{i}\right\}$ be a full set of mutually orthogonal primitive idempotents of $K(N)$. Since $K(N) L$ is an ideal we have a decomposition

$$
K(N) L=\sum \oplus K(N) L e_{i}
$$

Each $K(N) L e_{i}$ is indecomposable since $K(N) e_{i}$ has a unique minimal submodule. We also have $K(N) L e_{i} \neq(0)$ for the following reason. By (61.3) of [3] the dimension of a left ideal plus the dimension of its right annihilator is the dimension of the algebra. If $L_{0}$ is the annihilator of $L$ in $K(Q)$ then

$$
(K(N) L: K)+\left(L_{0} K(N): K\right)=[N: Q]\left\{(L: K)+\left(L_{0}: K\right)\right\}=|N|
$$

Hence $L_{0} K(N)=$ right annihilator of $K(N) L$. Since $Q \triangle N$ the ideal $L_{0} K(N)$ is in the radical of $K(N)$. In particular $L_{0} K(N)$ contains no idempotent $e_{i}$. 
Finally $K(N) e_{i} \cong K(N) e_{j}$ implies $K(N) L e_{i} \cong K(N) L e_{j}$ because $L K(N)=K(N) L$; $K(N) L e_{i} \cong K(N) L e_{j}$ implies $K(N) L e_{i}$ and $K(N) L e_{j}$ have isomorphic minimal submodules and hence $K(N) e_{i} \cong K(N) e_{j}$ by (58.12) of [3]. Hence the number of distinct indecomposable summands of $K(N) L$ is the number of distinct principal indecomposable $K(N)$-modules. By (83.5) of [3] this number is the number of $p$ regular conjugate classes of $N$. We denote this number by $n_{p}(N)$.

Since $Q$ is a normal subgroup of $N$, the Mackey formula gives $\left(L^{N}\right)_{Q} \cong[N: Q] \cdot L$. This shows that when $L$ has vertex $Q$ so does every indecomposable direct summand of $L^{N}$.

In case $Q=\langle 1\rangle$ there is one choice for $L$ and there are $n_{p}(G)$ indecomposable modules with vertex $Q$.

For $Q=\langle y\rangle \neq\langle 1\rangle$ the ideal $K(Q)(y-1)^{i}$ has vertex $Q$ if and only if it is not induced from a proper subgroup of $Q$. This holds if and only if $p$ does not divide $i$. Hence there are $|Q|(1-1 / p)$ ideals with vertex $Q$.

We summarize these remarks in the following result.

THEOREM. Let $G$ be a finite group with a cyclic p-Sylow subgroup $P$, and let $K$ be an algebraically closed field of characteristic $p$. The number of nonisomorphic indecomposable $K(G)$-modules is

$$
i(G)=n_{p}(G)+\sum|Q|(1-1 / p) n_{p}\left(N_{G}(Q)\right)
$$

where the sum is taken over all nonidentity subgroups $Q$ of $P$ and where $n_{p}(X)$ denotes the number of p-regular conjugate classes in the group $X$.

This can sometimes be useful when trying to compute the Cartan matrix $\left(c_{i j}\right)$ for $K(G),(83.8)$ of [3]. If $\left\{U_{i}\right\}$ is a full set of nonisomorphic principal indecomposable modules for $K(G)$ then the composition length of $U_{i}$ is $\sum_{j} c_{i j}$. Since every submodule of $U_{i}$ is indecomposable there are at least $\sum_{i, j} c_{i j}$ indecomposable $K(G)$-modules. This number will be the total number of indecomposable modules if and only if every indecomposable module has a unique simple submodule. By a theorem of Nakayama [8] this happens if and only if $K(G)$ is a generalized uniserial algebra. That is each $U_{i}$ has a unique composition series. So we have the following:

Corollary. Let $G, K$, and $i(G)$ be as in the theorem, and let $C=\left(c_{i j}\right)$ be the Cartan matrix for $K(G)$. Then $\sum_{i, j} c_{i j} \leqq i(G)$ with equality if and only if $K(G)$ is a generalized uniserial algebra.

3. Examples. Let $G=L F(2, p)$ for $p$ an odd prime. The order of $G$ is $\frac{1}{2}(p-1) p(p+1)$ and so the $p$-Sylow subgroup is cyclic of prime order $p$. Let $K$ be an algebraically closed field of characteristic $p$. We shall describe all the indecomposable representations of $G$ over $K$. The Cartan matrix for $K(G)$ is given in Brauer 
and Nesbitt [1]. There are $r+1=\frac{1}{2}(p+1)$ principal indecomposable modules $U_{0}, U_{1}, \ldots, U_{r}$. Let $F_{0}, F_{1}, \ldots, F_{r}$ be the corresponding irreducible modules. The composition factors of the $U_{i}$ are given as follows:

$$
\begin{aligned}
& U_{0}=F_{0} \quad U_{i} \sim 2 F_{i}+F_{i-1}+F_{i+1} \quad 1<i<r, \\
& U_{1} \sim 2 F_{1}+F_{2} \quad U_{r} \sim 3 F_{r}+F_{r-1} \text {. }
\end{aligned}
$$

To determine the indecomposable modules we must first determine the arrangement of the composition factors of the $U_{i}$. The following notation will be convenient. If $J=\operatorname{radical} K(G)$ and $M$ is a $K(G)$-module we write

$$
M \sim\left(i_{1}, \ldots, i_{s}\left|j_{1}, \ldots, j_{t}\right| \ldots\right)
$$

to indicate that

$$
M / J M \cong F_{i_{1}} \oplus \cdots \oplus F_{i_{s}}, \quad J M / J^{2} M \cong F_{j_{1}} \oplus \cdots \oplus F_{j_{t}}, \ldots
$$

From the properties of the injective hull of a module ( $\$ 57$ [3]) it follows that a module $M$ with a unique minimal submodule isomorphic to $F_{i}$ must be a submodule of $U_{i}$. Using this we determine that

$$
\begin{aligned}
U_{1} & \sim(1|2| 1), \\
U_{i} & \sim(i|i-1, i+1| i), \quad 1<i<r . \\
U_{r} & \sim(r|r-1, r| r),
\end{aligned}
$$

Let $V_{i}$ denote the unique maximal submodule of $U_{i}$. We arrange the $V_{i}$ according to the following scheme:

$$
\begin{array}{ll}
V_{1}, V_{3}, \ldots, V_{r-2}, V_{r}, V_{r-1}, V_{r-3}, \ldots, V_{2} & r \text { odd, } \\
V_{1}, V_{3}, \ldots, V_{r-1}, V_{r}, V_{r-2}, V_{r-4}, \ldots, V_{2} \quad r \text { even. }
\end{array}
$$

If $V_{a}$ immediately precedes $V_{b}$ in $\left(^{*}\right)$ then there is a unique index $d$ such that $V_{a}$ and $V_{b}$ have a common homomorphic image isomorphic to $F_{d}$. Let $\gamma_{a}, \lambda_{b}$ be homomorphisms such that $\gamma_{a}\left(V_{a}\right)=F_{d}=\lambda_{b}\left(V_{b}\right)$. Then $\gamma_{a}, \lambda_{b}$ are defined for $a \neq 2$ and $b \neq 1$.

Now let $V_{a}, \ldots, V_{k}$ be a collection of consecutive modules in the ordering (*) containing at least two $V$ 's. Let $M$ be the submodule of the direct sum $V_{a} \oplus \cdots \oplus V_{k}$ consisting of all tuples $\left(v_{a}, \ldots v_{k}\right)$ such that $\gamma_{s}\left(v_{s}\right)=\lambda_{t}\left(v_{t}\right)$ for all pairs $(s, t)$ for which $V_{s}$ immediately precedes $V_{t}$. We may repeat this construction to obtain $M^{\prime}$ by replacing $V_{k}$ with the smallest submodule of $V_{k}$ which is mapped onto $\lambda_{k}\left(V_{k}\right)$ by $\lambda_{k}$. If $V_{a} \neq V_{1}$ we may also replace $V_{a}$ with the smallest submodule of $V_{a}$ that maps onto $\gamma_{a}\left(V_{a}\right)$ under $\gamma_{a}$ to obtain $M^{\prime \prime}$. Finally if $V_{a} \neq V_{1}$ we can replace both $V_{a}$ and $V_{k}$ by appropriate submodules to get $M^{\prime \prime}$. We shall prove in the appendix 
that $M, M^{\prime}, M^{\prime \prime}, M^{\prime \prime \prime}$ are actually indecomposable. Observe that the socle of any of the modules here is the same as the socle of $V_{a} \oplus \cdots \oplus V_{k}$-namely $F_{a} \oplus \cdots \oplus F_{k}$. This is immediate because $\gamma_{s}\left(F_{s}\right)=0=\lambda_{t}\left(F_{t}\right)$. Hence the modules constructed in this way have different socles or else the same socles but different composition factors. So they are all different. Taking into account the $4+5(r-1)$ indecomposable submodules of the $U_{i}$ we have accounted for $\frac{1}{2}\left(p^{2}-p+2\right)$ indecomposable $K(G)$ modules. The formula of $\S 2$ assures us there are no others. For a discussion of the subgroups of $G$ see [2, Chapter 20].

It is interesting to note that the modules constructed are independent of the maps $\gamma_{a}, \lambda_{b}$.

Appendix. The purpose of this appendix is to discuss the subdirect sum of two modules over an arbitrary ring and give a test to determine when such a module is decomposable. In general subdirect sums of modules are very complicated to deal with. This case will be no exception. However when one of the modules in question is small enough, say with a unique minimal submodule, the test becomes manageable.

Let $A$ be an arbitrary ring and $M_{1}, M_{2}$ two left $A$-modules. Suppose there are homomorphisms $\varphi_{1}, \varphi_{2}$ of $M_{1}, M_{2}$ onto a common image $F$. We construct a submodule $M$ of $M_{1} \oplus M_{2}$ by taking all pairs $\left(m_{1}, m_{2}\right)$ for which $\varphi_{1}\left(m_{1}\right)=\varphi_{2}\left(m_{2}\right)$. Let $\pi_{i}$ be the projection of $M$ into $M_{i}$. We have $\pi_{i}(M)=M_{i}, i=1,2$.

Conversely suppose $M$ is a submodule of $M_{1} \oplus M_{2}$ with $\pi_{i}(M)=M_{i}$. Let $\pi_{i}$ be restricted to $M$ and set

$$
F=\pi_{1}(M) / \pi_{1}\left(\operatorname{ker} \pi_{2}\right) .
$$

Define $\varphi_{1}$ on $M_{1}$ to be the natural map $M_{1} \rightarrow F$. Let $\varphi_{2}$ be defined on $M_{2}$ by setting $\varphi_{2}\left(m_{2}\right)=\varphi_{1}\left(m_{1}\right)$ provided the pair $\left(m_{1}, m_{2}\right)$ is in $M$. By choice of $F, \varphi_{2}$ is welldefined and $M$ is the submodule of $M_{1} \oplus M_{2}$ constructed as above from $\varphi_{1}$ and $\varphi_{2}$. We use the notation $M=\left\{M_{i} ; \varphi_{i}\right\}$ for this module.

Now suppose $M=\left\{M_{i} ; \varphi_{i}\right\}$ is decomposable, say $M=L_{1} \oplus L_{2}$ with $L_{i} \neq(0)$. Then $L_{i} \subset M_{1} \oplus M_{2}$ so $L_{i}$ is a subdirect sum, not necessarily of $M_{1}, M_{2}$ but of $\pi_{j}\left(L_{i}\right)=M_{j i}$. There are maps $\lambda_{j i}$ such that $\lambda_{j i}\left(L_{i}\right)=E_{i}$ for some $E_{i}$ and

$$
L_{j}=\left\{M_{i j} ; \lambda_{i j}\right\} \quad 1 \leqq i, j \leqq 2 .
$$

Of course there are restrictions on the $\lambda_{i j}$ imposed by the fact that $L_{i} \subset M$. The precise relation is given in the following proposition.

Proposition. Let $M=\left\{M_{i} ; \varphi_{i}\right\} i=1,2$. Then $M$ is decomposable if and only if there exist modules $M_{i j}, E_{j}$ and maps $\lambda_{i j}, k_{i j}, \gamma_{j}$ for $i, j=1,2$ such that the following statements are true.

I. Every square in the diagram below is commutative.

II. $\lambda_{i j}\left(M_{i j}\right)=E_{j}$. 
III. For each $j, M_{1 j}$ and $M_{2 j}$ are not both the zero module.

IV. For each pair $\left(m_{1}, m_{2}\right)$ in $M$ there are uniquely determined pairs $\left(m_{1 j}, m_{2 j}\right)$ in $M_{1 j} \oplus M_{2 j}$ such that $\lambda_{1 j}\left(m_{1 j}\right)=\lambda_{2 j}\left(m_{2 j}\right)$ and $k_{i 1}\left(m_{i 1}\right)+k_{i 2}\left(m_{i 2}\right)=m_{i}$ for $i, j=1,2$.

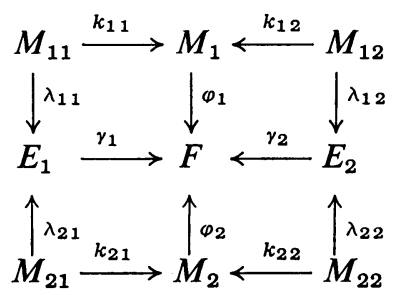

Diagram

Proof. Suppose I-IV hold. Let $L_{i}^{\prime}=\left\{M_{j i} ; \lambda_{j i}\right\}$ and $L_{i}=\left(k_{1 i} \oplus k_{2 i}\right) L_{i}^{\prime}$. By I, $L_{i} \subseteq M$ and $L_{i} \neq(0)$ by III. By IV we have $L_{1} \oplus L_{2} \cong M$.

Conversely suppose $M=L_{1} \oplus L_{2}$ with each $L_{i} \neq(0)$. Construct $M_{i j}, \lambda_{i j}$ as in the paragraph preceding the statement of the proposition. Let $k_{i j}$ be inclusions and define $\gamma_{i}$ so that the upper square commutes. That is for $m_{1 i} \in M_{1 i}$ let $\gamma_{i}\left(\lambda_{1 i}\left(m_{1 i}\right)\right)=\varphi_{1}\left(k_{1 i}\left(m_{1 i}\right)\right)$. Suppose $\lambda_{11}\left(m_{11}\right)=0$. Then there is an element $\left(m_{11}, 0\right)$ in $L_{1}$ and hence in $M$. Thus $\varphi_{1}\left(m_{11}\right)=0$ so $\gamma_{1}$ is well defined. Similarly $\gamma_{2}$ is well defined. We now check that the lower squares commute. Pick $m_{21} \in M_{21}$. There is an element $\left(m_{11}, m_{21}\right)$ in $L_{1}$ for which $\lambda_{11}\left(m_{11}\right)=\lambda_{21}\left(m_{21}\right)$. Thus

$$
\gamma_{1} \circ \lambda_{21}\left(m_{21}\right)=\gamma_{1} \circ \lambda_{11}\left(m_{11}\right)=\varphi_{1} \circ k_{11}\left(m_{11}\right)=\varphi_{1}\left(m_{11}\right) \text {. }
$$

The element $\left(m_{11}, m_{21}\right)$ must also belong to $M$ so $\varphi_{1}\left(m_{11}\right)=\varphi_{2}\left(m_{21}\right)$. Thus

$$
\gamma_{2} \circ \lambda_{21}\left(m_{21}\right)=\varphi_{2}\left(m_{21}\right)
$$

as required. Use a similar argument for the lower right hand square. This proves $I$. Statement II follows from the construction. The fact that $L_{i} \neq(0)$ implies III and statement IV follows because the sum $L_{1} \oplus L_{2}$ is a direct sum.

It may be difficult to use this proposition because there is no indication how one should choose the $M_{i j}$ having been given only $\left\{M_{i} ; \varphi_{i}\right\}$. We list three facts which may help to determine the $M_{i j}$ in certain cases. Again consider the $k_{i j}$ as inclusions.

$$
\begin{aligned}
& M_{i 1}+M_{i 2}=M_{i} \quad \text { for } i=1,2 . \\
& \operatorname{ker} \lambda_{i 1} \cap \operatorname{ker} \lambda_{i 2}=(0) \quad \text { for } i=1,2 . \\
& \gamma_{1}\left(E_{1}\right)+\gamma_{2}\left(E_{2}\right)=F
\end{aligned}
$$

Statement (2) follows because a nonzero element in the intersection can be used to violate the uniqueness in IV for the element $\left(m_{1}, m_{2}\right)=(0,0)$.

We now apply this proposition to show the modules constructed in the previous section are indecomposable. If any of the modules can be decomposed then there is a smallest one that can be decomposed. Let $V_{a}, \ldots, V_{e}, V_{k}$ be the shortest sequence 
in the ordering $\left(^{*}\right)$ for which one of the four (or possibly two if $V_{a}=V_{1}$ ) modules $M, M^{\prime}, M^{\prime \prime}, M^{m}$ is decomposable. We suppose $M$ is the one, the treatment of the other cases being almost exactly the same.

In order to apply the proposition we first describe $M$ as a subdirect sum of two modules, $M_{1}$ and $M_{2}$. For $M_{1}$ take $V_{k}$ and for $\varphi_{1}$ take $\lambda_{k}$. For $M_{2}$ take the module constructed from the sequence $V_{a}, \ldots, V_{e}$ (or take $M_{2}=V_{a}$ if $V_{e}=V_{a}$ ) and for $\varphi_{2}$ take the map $\gamma_{e} \circ \pi_{e}$ where $\pi_{e}$ is the projection onto $V_{e}$. Then we have $M=\left\{M_{i} ; \varphi_{i}\right\}$. We suppose $M=L_{1} \oplus L_{2}$ with $L_{i} \neq(0)$ and so we have a collection of modules and maps as given in the proposition and the diagram such that I-IV are valid.

$M_{1}$ has a unique minimal submodule so nonzero submodules have a nonzero intersection. Thus by statement (2) one of the maps $\lambda_{11}, \lambda_{12}$ is one-to-one. Suppose it is $\lambda_{12}$. Then $E_{2}$ is a submodule of $M_{1}$ and also a homomorphic image of a submodule of $M_{2}$. By inspecting the composition factors of $M_{2}$ we see there cannot be a submodule $E_{2}$ of $M_{1}$ which is a homomorphic image of a submodule of $M_{2}$ and which also maps onto $F$. Thus $\gamma_{2}\left(E_{2}\right)=(0)$ and $M_{22} \subseteq \operatorname{ker} \varphi_{2}$.

If we also take into account the arrangement of the composition factors of $M_{2}$ we see that $E_{2}$ must be $F_{k}$ (the minimal submodule of $M_{1}$ ) or (0), for no other submodule is a homomorphic image of a submodule of ker $\varphi_{2}$. Since $\lambda_{12}$ is one-toone we have $M_{12}=F_{k}$ or $M_{12}=(0)$. In either case statement (1) above implies $M_{11}=M_{1}$. Since no submodule of $M_{2}$ can map onto $M_{1}$ we see $\lambda_{11}$ is not one-toone. In particular

$$
F_{k} \subseteq \operatorname{ker} \lambda_{11}
$$

Now take any $m_{2} \in \operatorname{ker} \varphi_{2}$. By IV we have

$$
\left(0, m_{2}\right)=\left(m_{11}, m_{21}\right)+\left(-m_{11}, m_{22}\right) \text {. }
$$

The element $-m_{11}$ is in $M_{12} \subseteq F_{k}$ and so by (4) $\lambda_{11}\left(m_{11}\right)=(0)$. By the condition in IV, $\lambda_{21}\left(m_{21}\right)=0$. This proves

$$
\operatorname{ker} \varphi_{2}=\operatorname{ker} \lambda_{21}+M_{22}
$$

Now choose $z \in \operatorname{ker} \lambda_{21} \cap M_{22}$. There is some $m_{12} \in F_{k}$ such that $\left(m_{12}, z\right) \in L_{2}$. By (4), $\lambda_{11}\left(m_{12}\right)=0=\lambda_{21}(z)$ so that $\left(m_{12}, z\right) \in L_{1}$. Since $L_{1} \oplus L_{2}$ is a direct sum we have $z=0$. In particular (5) is a direct sum decomposition of $\operatorname{ker} \varphi_{2}$.

However $\operatorname{ker} \varphi_{2}$ is a module of the type $M^{\prime}$ constructed from the sequence $V_{a}, \ldots, V_{e}$. By the choice of $M$ we must have $\operatorname{ker} \varphi_{2}$ indecomposable. Hence either ker $\lambda_{21}=(0)$ or $M_{22}=(0)$.

Consider the first choice. If $\lambda_{21}$ is one-to-one, then $M_{21}$ is a homomorphic image of $M_{1}$ (= $\left.=M_{11}\right)$ with kernel containing $F_{k}$. Thus $M_{21}$ is completely reducible. If $M_{21}=U \oplus\left(M_{21} \cap M_{22}\right)$ then by statement (1) we have $M_{2}=U \oplus M_{22}$. Again our choice of $M$ implies $M_{2}$ is indecomposable. If $U=0$ then $M_{2}=M_{22} \subseteq \operatorname{ker} \varphi_{2}$. 
This is impossible. If $M_{22}=(0)$ then $E_{2}=(0)$ and $M_{12}=(0)$ since $\lambda_{12}$ is one-to-one. But this violates III in the proposition. Thus ker $\lambda_{21} \neq(0)$ so $M_{22}=(0)$. But we have just seen $M_{22}=(0)$ gives a contradiction. Hence the assumption that $M$ is decomposable is not valid.

\section{REFERENCES}

1. R. Brauer and C. Nesbitt, On the modular characters of groups, Ann. of Math. (2) 42 (1941), 556-590.

2. W. Burnside, Theory of groups of finite order, 2nd ed., Cambridge Univ. Press, Cambridge, 1911.

3. C. W. Curtis and I. Reiner, Representation theory of finite groups and associative algebras, Interscience, New York, 1962.

4. J. A. Green, On the indecomposable representations of a finite group, Math. Z. 70 (1959), $430-445$.

5. — A transfer theorem for modular representations, J. Algebra 1 (1964), 73-84.

6. D. G. Higman, Indecomposable representations at characteristic p, Duke Math. J. 21 (1954), 377-381.

7. F. Kasch, M. Kneser and H. Kupisch, Unzerlegbare modulare Darstellungen endlicher Gruppen mit zyklischer p-Sylow-Gruppe, Arch. Math. 8 (1957), 320-321.

8. T. Nakayama, On Frobenius algebras. II, Ann. of Math. 42 (1941), 1-21.

INSTITUTE for AdVANCEd STUdy,

Princeton, NeW Jersey 